\title{
SELF-CALIBRATION OF THE 1 MN DEADWEIGHT FORCE STANDARD MACHINE AT INRIM
}

\author{
A. Prato ${ }^{1}$, F. Mazzoleni ${ }^{1}$, A. Facello ${ }^{1}$, A. Germak ${ }^{1}$ \\ ${ }^{1}$ INRiM - Istituto Nazionale di Ricerca Metrologica, Torino, Italy, a.prato@inrim.it
}

\begin{abstract}
:
The INRiM $1 \mathrm{MN}$ deadweight force standard machine (DFSM) was installed in 1995. It adopts a binary sequence of ten weights whose combinations generate forces up to $1 \mathrm{MN}$. The advantage of this system lies in the self-calibration of its weights. The procedure is based on the comparison between two forces generated by a single weight and by a group of smaller weights, nominally equal. After 25 years, a verification of the DFSM was performed. Results are within the declared CMC limits, i.e. a relative expanded uncertainty of $2 \times 10^{-5}$.
\end{abstract}

Keywords: deadweight force standard machine; self-calibration; uncertainty

\section{INTRODUCTION}

Back in 1995, the advancement of technology and available resources allowed to design and install the $1 \mathrm{MN}$ DFSM at INRiM $[1,2]$. The machine is able to generate known forces and is generally used as a reference for the calibration or verification of force transducers and load cells. The DFSM has a declared CMC with a relative expanded uncertainty of $2 \times 10^{-5}$ [3]. Twenty-five years after the installation, despite several positive international comparisons, it was necessary to carry out a verification of the machine. For this purpose, a self-calibration method was developed. Measurements were performed between November 2018 and April 2019. This paper deals with the description of the self-calibration method and the analysis of experimental results.

\section{DESCRIPTION OF THE 1 MN DEADWEIGHT FORCE MACHINE}

The 1 MN DFSM was designed to obtain a reference standard capable of keeping its metrological characteristics unchanged. It consists of three macro-components: a main frame that supports the entire structure, a loading frame, and a series of weights acting in the local gravitational field. The main frame consists of three columns anchored to the load-bearing structure arranged at $120^{\circ}$ on a diameter of about $6 \mathrm{~m}$ (Figure 1 ).
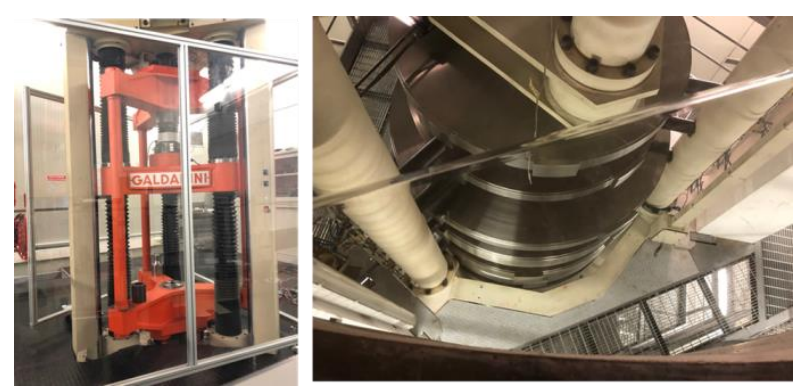

Figure 1: The $1 \mathrm{MN}$ deadweight force standard machine

The 1 MN DFSM adopts a binary sequence of ten weights (two $10 \mathrm{kN}$ weights, three weights of $20 \mathrm{kN}, 40 \mathrm{kN}$, and $80 \mathrm{kN}$, four weights of $160 \mathrm{kN}$, and one weight of $200 \mathrm{kN}$ ), consisting of stainless steel discs. Each of them can be applied to the loading frame independently to the others. In this way, it is possible to generate a large number of force values from $10 \mathrm{kN}$ up to a maximum capacity of $1 \mathrm{MN}$ with steps of $10 \mathrm{kN}$, and to perform a self-calibration of the weights to check the stability of the standard without dismantling the whole system [4]. The selection of the weights is performed by an electro-pneumatic system, software controlled, and supported by an electric motor able to generate a constant load on the force transducer during weights replacement operations. The load is kept constant by a feedback system exploiting the deformation elasticity of the loading frame.

\section{SELF-CALIBRATION PROCEDURE}

The self-calibration method is based on the comparison between two nominally equal forces alternatively generated by a single weight and by an equivalent group of smaller weights [5]. The reference force transducers adopted for the selfcalibration were a TOP-Transfer HBM (Z30A and Z4A), with HBM DMP40 amplifier. The initial reference force was provided by the smallest weight, associated with a force of $10 \mathrm{kN}$ (M10/1), which was previously calibrated against a reference mass 
standard before the installation of the machine. Another advantage of this method is that it can consider any influences due to the behaviour of the whole structure under load since it directly compares the force vectors applied on the force transducer and not the force generated in centre of mass as in the standard method for calibrated force standard machines. For each force value $(10 \mathrm{kN}$, $20 \mathrm{kN}, \quad 40 \mathrm{kN}, \quad 80 \mathrm{kN}, 160 \mathrm{kN}, 200 \mathrm{kN}$ ), two identical forces were alternately generated: one generated by the single nominal weight (Measure B), the other by the sum of smaller weights of equivalent total force (Measure A). The forces generated for each weight comparison are shown in Table 1.

Table 1: Weights used for the comparison

\begin{tabular}{|l|c|l|}
\hline Weight & $\begin{array}{c}\text { Single weight } \\
\text { force / kN } \\
\text { (Measure B) }\end{array}$ & $\begin{array}{c}\text { Combination of } \\
\text { smaller weights with } \\
\text { equivalent force } \\
\text { (Measure A) }\end{array}$ \\
\hline M10/1 & 10 & - \\
\hline M10/2 & 10 & M10/1 \\
\hline M20 & 20 & M10/1 + M10/2 \\
\hline M40 & 40 & M10/1+ M10/2+ M20 \\
\hline M80 & 80 & $\begin{array}{l}\text { M10/1 + M10/2+ M20 } \\
+\mathrm{M} 40\end{array}$ \\
\hline M160/1 & 160 & M160/4 \\
\hline M160/2 & 160 & M160/4 \\
\hline M160/3 & 160 & M160/4 \\
\hline M160/4 & 160 & $\begin{array}{l}\text { M10/1 + M10/2 + M20 } \\
+ \text { M40 + M80 }\end{array}$ \\
\hline M200 & 200 & M160/4 + M40 \\
\hline
\end{tabular}

A comparison scheme was then adopted to identify an ABAB...BA type sequence. This sequence was chosen since the most significant causes of disturbance are due to weights replacement operations and to creep effects of the force transducers. For each pair of equal forces, $j=10$ measurement runs were performed. During each $j$-th run, $i=20$ force values were acquired in a time interval of $20 \mathrm{~s}$.

\section{ANALYSIS OF RELATIVE DEVIATIONS}

Once measurements were performed, data analysis was carried out for each force value. First, the average, corrected by subtracting the zero value, of the $i$-th temporal acquisition values of the $j$-th run, $F_{a, i j}$ and $F_{b, i j}$, related to the two forces alternately generated and nominally equal $\left(F_{a, i j}\right.$ corresponds to the force generated by the reference $10 \mathrm{kN}$ weight, M10/1, or by the sum of the smaller weights for forces greater than or equal to $20 \mathrm{kN}$, and $F_{b, i j}$ corresponds to the force generated by the single weight), was performed according to equation (1).
The average of the temporal mean values $\overline{F_{a, j}}$ and $\overline{F_{b, J}}$ of the 10 runs is given by equation (2).

$\overline{F_{a, j}}=\sum_{i=1}^{20} \frac{F_{a, i j}}{20}$

$\overline{F_{b, J}}=\sum_{i=1}^{20} \frac{F_{b, i j}}{20}$

$\bar{F}=\sum_{j=1}^{10} \frac{\left(\overline{F_{a, j}}+\overline{F_{b, j}}\right)}{2}=\sum_{j=1}^{10} \frac{\overline{F_{a, j}}+\overline{F_{b, j}}}{20}$

To minimize creep effect, although the short time elapsed between two replacements (about $100 \mathrm{~s})$, a processing scheme, based on the differences between a single value and the mean value between the previous and next measurement, was used. According to this scheme, the absolute deviations $d_{j}$, equation (3) and relative deviations $\delta_{j}$, equation (4), were calculated for each force value $F$.

$d_{a, j}=\frac{\overline{F_{a, j}}+\overline{F_{a, J+1}}}{2}-\overline{F_{b, J}}$

$d_{b, j}=\overline{F_{a, j}}-\frac{\overline{F_{b, j}}+\overline{F_{b, J+1}}}{2}$

$\delta_{a, j}=\frac{d_{a, j}}{\bar{F}}$

$\delta_{b, j}=\frac{d_{b, j}}{\bar{F}}$

In this way, it was possible to calculate the mean relative deviation $\bar{\delta}$ according to equation (5).

$\bar{\delta}=\frac{\sum_{j=1}^{10} \frac{d_{a, j}+d_{b, j}}{20}}{\bar{F}}=\sum_{j=1}^{10} \frac{\delta_{a, j}+\delta_{b, j}}{20}$

To check the actual force values generated by the individual weights, it was necessary to add the mean relative deviations $\bar{\delta}$ of the smaller weights used. In this way, the total mean relative deviations $\bar{\Delta}$ can be obtained. Assuming that the force $F_{\mathrm{M} 10 / 1}$ generated by the reference M10/1 weight is exactly $10 \mathrm{kN}$ with relative expanded uncertainty of $2.82 \times 10^{-6}[6]$, the mean relative deviation of the reference $10 \mathrm{kN}$ weight (M10/1), $\bar{\delta}_{\mathrm{M} 10 / 1}$, is equal to zero, with an associated uncertainty equal to the uncertainty of the reference force $U\left(\bar{\delta}_{\mathrm{M} 10 / 1}\right)=U\left(F_{\mathrm{M} 10 / 1}\right) / F_{\mathrm{M} 10 / 1}=2.82 \times 10^{-6}$. In this way, equations (6) and (7) are obtained. 
$\bar{\delta}_{\mathrm{M} 10 / 1}=\bar{\Delta}_{\mathrm{M} 10 / 1}=\frac{F_{\mathrm{M} 10 / 1}-10}{10}=0$

$\frac{F_{\mathrm{M} 10 / 1}}{10}=1+\bar{\delta}_{\mathrm{M} 10 / 1}$

As a consequence, the mean relative deviation of M10/2 weight, $\bar{\delta}_{\mathrm{M} 10 / 2}$, can be written as

$$
\begin{aligned}
& \bar{\delta}_{\mathrm{M} 10 / 2}=\frac{F_{\mathrm{M} 10 / 1}-F_{\mathrm{M} 10 / 2}}{10}= \\
& =1+\bar{\delta}_{\mathrm{M} 10 / 1}-\frac{F_{\mathrm{M} 10 / 2}}{10}
\end{aligned}
$$

from which, the total relative deviation of weight $\mathrm{M} 10 / 2, \bar{\Delta}_{\mathrm{M} 10 / 2}$, is obtained:

$$
\bar{\Delta}_{\mathrm{M} 10 / 2}=\frac{F_{\mathrm{M} 10 / 2}-10}{10}=\bar{\delta}_{\mathrm{M} 10 / 1}-\bar{\delta}_{\mathrm{M} 10 / 2}
$$

By operating in the same way for weight M20, the following relations are obtained:

$$
\begin{aligned}
& \bar{\delta}_{\mathrm{M} 20}=\frac{F_{\mathrm{M} 10 / 1}+F_{\mathrm{M} 10 / 2}-F_{\mathrm{M} 20}}{20}= \\
& =\frac{1}{2} \frac{F_{\mathrm{M} 10 / 1}}{10}+\frac{1}{2} \frac{F_{\mathrm{M} 10 / 2}}{10}-\frac{F_{\mathrm{M} 20}}{20}= \\
& =1+\bar{\delta}_{\mathrm{M} 10 / 1}-\frac{1}{2} \bar{\delta}_{\mathrm{M} 10 / 2}-\frac{F_{\mathrm{M} 20}}{20} \\
& \bar{\Delta}_{\mathrm{M} 20}=\frac{F_{\mathrm{M} 20}-20}{20}= \\
& =\bar{\delta}_{\mathrm{M} 10 / 1}-\frac{1}{2} \bar{\delta}_{\mathrm{M} 10 / 2}-\bar{\delta}_{\mathrm{M} 20}
\end{aligned}
$$

Iterating the same procedure for all other weights, the following equations are obtained:

$$
\begin{aligned}
& \bar{\delta}_{\mathrm{M} 40}=\frac{F_{\mathrm{M} 10 / 1}+F_{\mathrm{M} 10 / 2}+F_{\mathrm{M} 20}-F_{\mathrm{M} 40}}{40} \\
& \bar{\Delta}_{\mathrm{M} 40}=\frac{F_{\mathrm{M} 40}-40}{40}= \\
& =\bar{\delta}_{\mathrm{M} 10 / 1}-\frac{1}{2} \bar{\delta}_{\mathrm{M} 10 / 2}-\frac{1}{2} \bar{\delta}_{\mathrm{M} 20}-\bar{\delta}_{\mathrm{M} 40} \\
& \bar{\delta}_{\mathrm{M} 80}= \\
& =\frac{F_{\mathrm{M} 10 / 1}+F_{\mathrm{M} 10 / 2}+F_{\mathrm{M} 20}+F_{\mathrm{M} 40}-F_{\mathrm{M} 80}}{80} \\
& \bar{\Delta}_{\mathrm{M} 80}=\frac{F_{\mathrm{M} 80}-80}{80}= \\
& =\bar{\delta}_{\mathrm{M} 10 / 1}-\frac{1}{2} \bar{\delta}_{\mathrm{M} 10}-\frac{1}{2} \bar{\delta}_{\mathrm{M} 20}-\frac{1}{2} \bar{\delta}_{\mathrm{M} 40}- \\
& -\bar{\delta}_{\mathrm{M} 80}
\end{aligned}
$$

$\bar{\Delta}_{\mathrm{M} 200}=\frac{F_{\mathrm{M} 200}-200}{200}=$

$=\bar{\delta}_{\mathrm{M} 10 / 1}-\frac{1}{2} \bar{\delta}_{\mathrm{M} 10 / 2}-\frac{1}{2} \bar{\delta}_{\mathrm{M} 20}-\frac{3}{5} \bar{\delta}_{\mathrm{M} 40}-$

$-\frac{2}{5} \bar{\delta}_{\mathrm{M} 80}-\frac{4}{5} \bar{\delta}_{\mathrm{M} 160 / 4}-\bar{\delta}_{\mathrm{M} 200}$ 


\section{UNCERTAINTY ASSESSMENT}

The uncertainty analysis was carried out according to GUM-JCGM 100:2008 [7]. For each force value, the uncertainty analysis of the total mean relative deviation $\bar{\Delta}$ was carried out in two consecutive steps. In the first, the uncertainty associated to the mean relative deviations $\bar{\delta}$ of the single weight comparisons was assessed, taking into account the maximum uncertainty among the time series of twenty measurements, corresponding to the different weights substitutions used for the evaluation of the relative deviations $\delta_{j}$, and the reproducibility uncertainty contribution due to the ten measurement runs. In the second, by applying the law of propagation of errors, the expanded uncertainty, at a confidence level of $95 \%$, of the total mean relative deviations $\bar{\Delta}$ was evaluated.

\subsection{Uncertainty of Mean Relative Deviations}

First, among the $j$-th relative deviations $\delta_{j}$, the one with maximum uncertainty due to repeatability of each force measurement of the three successive time series $\left(\overline{F_{a, j}}, \overline{F_{b, j}}, \overline{F_{a, j+1}}\right.$ or $\left.\overline{F_{b, j}}, \overline{F_{a, j}}, \overline{F_{b, J+1}}\right)$ was evaluated. Once the $j$-th relative deviation $\delta_{j}$ with the maximum dispersione was identified, the uncertainty contribution due to the resolution of the HBM control unit was added for each force value. No uncertainty was associated to the value $\bar{F}$, since it was used only to calculate the relative difference from absolute measurements. By way of example, the detailed uncertainty budget for $\delta_{j}$ from the comparison between the $10 \mathrm{kN}$ weights (M10/1 and $\mathrm{M} 10 / 2$ ) is shown in Table 2. Force values are expressed in $\mathrm{mV} / \mathrm{V}$.

Table 2: Uncertainty budget of the $j$-th relative deviation $\delta_{j}$ with maximum dispersion from the comparison between the $10 \mathrm{kN}$ weights $\left(\bar{\delta}_{\mathrm{M} 10 / 2}\right)$

\begin{tabular}{|c|c|c|c|c|c|}
\hline \multicolumn{3}{|c|}{ Variable $x_{k}$} & \multirow{2}{*}{$u^{2}\left(x_{k}\right)$} & \multirow{2}{*}{$c_{k}$} & \multirow{2}{*}{$\boldsymbol{u}_{k}^{2}\left(a_{x}\right)$} \\
\hline Symbol & Value & Note & & & \\
\hline \multirow{2}{*}{$\overline{F_{a, j}}$} & 0.999494 & Res. & $1.7 \mathrm{E}-14$ & 0.5 & $4.2 \mathrm{E}-15$ \\
\hline & & Repeat. & $4.9 \mathrm{E}-14$ & 0.5 & $1.2 \mathrm{E}-14$ \\
\hline \multirow[t]{2}{*}{$\overline{F_{b, j}}$} & 0.999500 & Res. & $1.7 \mathrm{E}-14$ & 1.0 & $1.7 \mathrm{E}-14$ \\
\hline & & Repeat. & $1.2 \mathrm{E}-12$ & 1.0 & $1.2 \mathrm{E}-12$ \\
\hline \multirow[t]{2}{*}{$\overline{F_{a, j+1}}$} & 0.999499 & Res. & $1.7 \mathrm{E}-14$ & 0.5 & 4.2E-15 \\
\hline & & Repeat. & $1.6 \mathrm{E}-13$ & 0.5 & $3.9 \mathrm{E}-14$ \\
\hline $\bar{F}$ & 0.999490 & - & - & - & - \\
\hline \multirow[t]{2}{*}{$\delta_{a, j}$} & $-3.33 \mathrm{E}-06$ & & nance, & $\left(\delta_{a, j}\right)$ & $1.3 \mathrm{E}-12$ \\
\hline & & adard $u$ & rtainty, & $\left(\delta_{a, j}\right)$ & $1.1 \mathrm{E}-06$ \\
\hline
\end{tabular}

Subsequently, the evaluation of the expanded uncertainty associated with the mean relative deviation $U(\bar{\delta})$ was performed, as shown in Table 3. The variance of the relative deviation, $u^{2}\left(\delta_{j}\right)$, obtained from Table 2 (in bold), was used as variance associated with the maximum standard deviation. As uncertainty contribution due to reproducibility, the standard deviation of the ten measurement runs $\delta_{a j}$ and $\delta_{b j}$ was considered. The expanded uncertainty $U(\bar{\delta})$ of the mean relative deviations was calculated at a confidence level of $95 \%$, i.e. $k=2$. For each force value, the same procedure was repeated by assessing reproducibility and maximum standard deviation of the temporal series. Results of each weight comparison are shown in Section 6.

Table 3: Uncertainty budget of the mean relative deviation $\bar{\delta}$ from the comparison between the $10 \mathrm{kN}$ weights

\begin{tabular}{|c|c|c|c|c|c|}
\hline \multicolumn{3}{|c|}{ Variable $x_{k}$} & \multirow{2}{*}{$u^{2}\left(x_{k}\right)$} & \multirow{2}{*}{$c_{k}$} & \multirow{2}{*}{$u_{k^{2}}^{2}\left(a_{x}\right)$} \\
\hline Symbol & Value & Note & & & \\
\hline \multirow[t]{2}{*}{$\bar{\delta}$} & $-2.49 \mathrm{E}-06$ & Reprod. & $2.0 \mathrm{E}-13$ & 1.0 & $2.0 \mathrm{E}-13$ \\
\hline & & $\begin{array}{l}\text { Max. } \\
\text { st. dev. }\end{array}$ & $1.3 \mathrm{E}-12$ & 1.0 & $1.3 \mathrm{E}-12$ \\
\hline & $-2.49 \mathrm{E}-06$ & & Variance, & $u^{2}(\bar{\delta})$ & 1.5E-12 \\
\hline & & Standard $\mathrm{u}$ & incertainty & $u(\bar{\delta})$ & $1.2 \mathrm{E}-06$ \\
\hline
\end{tabular}

\subsection{Uncertainty of Total Mean Relative Deviations}

By applying the law of propagation of errors to Eqs. (6), (9), (11), (13), (15), (17), (19), (21), (23), and (25), using as input the expanded uncertainties associated with the mean relative deviations $U(\delta)$ evaluated according to Section 5.1, the expanded uncertainties of the total relative deviations $U(\bar{\Delta})$ are obtained. By way of example, the uncertainties for the M10/2, M20, and M40 are reported in equations (26) - (28).

$$
\begin{aligned}
& U\left(\bar{\Delta}_{\mathrm{M} 10 / 2}\right)= \\
& =2 \sqrt{\left(\frac{U\left(\bar{\delta}_{\mathrm{M} 10 / 1}\right)}{2}\right)^{2}+\left(\frac{U\left(\bar{\delta}_{\mathrm{M} 10 / 2}\right)}{2}\right)^{2}} \\
& U\left(\bar{\Delta}_{\mathrm{M} 20}\right)= \\
& =2 \sqrt{\left(\frac{U\left(\bar{\delta}_{\mathrm{M} 10 / 1}\right)}{2}\right)^{2}+\left(\frac{1}{2}\right)^{2}\left(\frac{U\left(\bar{\delta}_{\mathrm{M} 10 / 2}\right)}{2}\right)^{2}+} \\
& +\left(\frac{U\left(\bar{\delta}_{\mathrm{M} 20}\right)}{2}\right)^{2}
\end{aligned}
$$

$$
\begin{aligned}
& U\left(\bar{\Delta}_{\mathrm{M} 40}\right)= \\
& =2 \sqrt{\left(\frac{U\left(\bar{\delta}_{\mathrm{M} 10 / 1}\right)}{2}\right)^{2}+\left(\frac{1}{2}\right)^{2}\left(\frac{U\left(\bar{\delta}_{\mathrm{M} 10 / 2}\right)}{2}\right)^{2}+} \\
& +\left(\frac{1}{2}\right)^{2}\left(\frac{U\left(\bar{\delta}_{\mathrm{M} 20}\right)}{2}\right)^{2}+\left(\frac{U\left(\bar{\delta}_{\mathrm{M} 40}\right)}{2}\right)^{2}
\end{aligned}
$$




\section{EXPERIMENTAL RESULTS}

\subsection{Mean Relative Deviations $\bar{\delta}$}

Overall, nine mean relative deviations $\bar{\delta}$, were calculated, each referred to a particular force value (or weight comparison), according to Table 1. Summary of experimental results is shown in Table 4.

Table 4: Mean relative deviations

\begin{tabular}{|l|c|c|c|}
\hline Weight & $\boldsymbol{F} / \mathbf{k N}$ & $\overline{\boldsymbol{\delta}} / \boldsymbol{-}$ & $\boldsymbol{U}(\overline{\boldsymbol{\delta}}) / \boldsymbol{-}$ \\
\hline $\mathrm{M} 10 / 1$ & 10 & $0.00 \mathrm{E}+00$ & $2.82 \mathrm{E}-06$ \\
\hline $\mathrm{M} 10 / 2$ & 10 & $-2.49 \mathrm{E}-06$ & $2.55 \mathrm{E}-06$ \\
\hline M20 & 20 & $-1.23 \mathrm{E}-05$ & $5.38 \mathrm{E}-06$ \\
\hline $\mathrm{M} 40$ & 40 & $-4.46 \mathrm{E}-06$ & $6.54 \mathrm{E}-07$ \\
\hline M80 & 80 & $8.02 \mathrm{E}-06$ & $3.92 \mathrm{E}-06$ \\
\hline $\mathrm{M} 160 / 1$ & 160 & $-2.13 \mathrm{E}-06$ & $8.09 \mathrm{E}-07$ \\
\hline $\mathrm{M} 160 / 2$ & 160 & $-3.31 \mathrm{E}-07$ & $3.86 \mathrm{E}-07$ \\
\hline M160/3 & 160 & $-3.51 \mathrm{E}-06$ & $6.15 \mathrm{E}-07$ \\
\hline M160/4 & 160 & $-3.19 \mathrm{E}-06$ & $6.36 \mathrm{E}-07$ \\
\hline M200 & 200 & $2.03 \mathrm{E}-06$ & $2.57 \mathrm{E}-07$ \\
\hline
\end{tabular}

\subsection{Total Mean Relative Deviations $\bar{\Delta}$}

Using data of Table 4 and equations (6) - (28), the total mean relative deviations $\bar{\Delta}$ of all weights are obtained with the associated expanded uncertainties. Results show values lower than the limits declared in CMC, i.e. within an expanded uncertainty of $2 \times 10^{-5}$, as reported in Table 5 and in Figure 2.
Table 5: Total mean relative deviations of the weights

\begin{tabular}{|l|c|c|c|}
\hline Weight & $\boldsymbol{F} / \mathbf{k N}$ & $\overline{\boldsymbol{\Delta}} / \boldsymbol{-}$ & $\boldsymbol{U}(\overline{\boldsymbol{\Delta}}) / \boldsymbol{-}$ \\
\hline $\mathrm{M} 10 / 1$ & 10 & $0.00 \mathrm{E}+00$ & $2.82 \mathrm{E}-06$ \\
\hline $\mathrm{M} 10 / 2$ & 10 & $2.49 \mathrm{E}-06$ & $3.80 \mathrm{E}-06$ \\
\hline $\mathrm{M} 20$ & 20 & $1.35 \mathrm{E}-05$ & $6.21 \mathrm{E}-06$ \\
\hline $\mathrm{M} 40$ & 40 & $1.18 \mathrm{E}-05$ & $4.15 \mathrm{E}-06$ \\
\hline $\mathrm{M} 80$ & 80 & $1.59 \mathrm{E}-06$ & $5.68 \mathrm{E}-06$ \\
\hline $\mathrm{M} 160 / 1$ & 160 & $6.65 \mathrm{E}-06$ & $4.67 \mathrm{E}-06$ \\
\hline $\mathrm{M} 160 / 2$ & 160 & $8.45 \mathrm{E}-06$ & $4.62 \mathrm{E}-06$ \\
\hline $\mathrm{M} 160 / 3$ & 160 & $5.27 \mathrm{E}-06$ & $4.64 \mathrm{E}-06$ \\
\hline $\mathrm{M} 160 / 4$ & 160 & $8.78 \mathrm{E}-06$ & $4.60 \mathrm{E}-06$ \\
\hline $\mathrm{M} 200$ & 200 & $7.36 \mathrm{E}-06$ & $4.44 \mathrm{E}-06$ \\
\hline
\end{tabular}

6.3. Total Relative Deviations $\bar{\Delta}$ of Typical Weight Combinations Used for the Calibration of Force Transducers

Finally, the total mean relative deviations of different force combinations were assessed. In fact, during ordinary calibration procedures of force transducers, according to UNI EN ISO 376:2011 [8], the $1 \mathrm{MN}$ DFSM adopts predefined series of weights able to generate the required different force values.

For this purpose, the total absolute deviations $\bar{\Delta}$ of each weight, previously shown, were combined in order to obtain the total relative deviations for each force generated by any combination of weights, with the associated expanded uncertainty calculated using the law of propagation of errors as in equations (26) - (28). Also in this case, results fall within the limits of the declared CMCs [3] as shown in the graph of Figure 3. Furthermore, $500 \mathrm{kN}$ and $1 \mathrm{MN}$ total relative deviations, which are around $7 \mathrm{ppm}-8 \mathrm{ppm}$, confirm results of Force Key Comparison CCM.F-K3 [9].

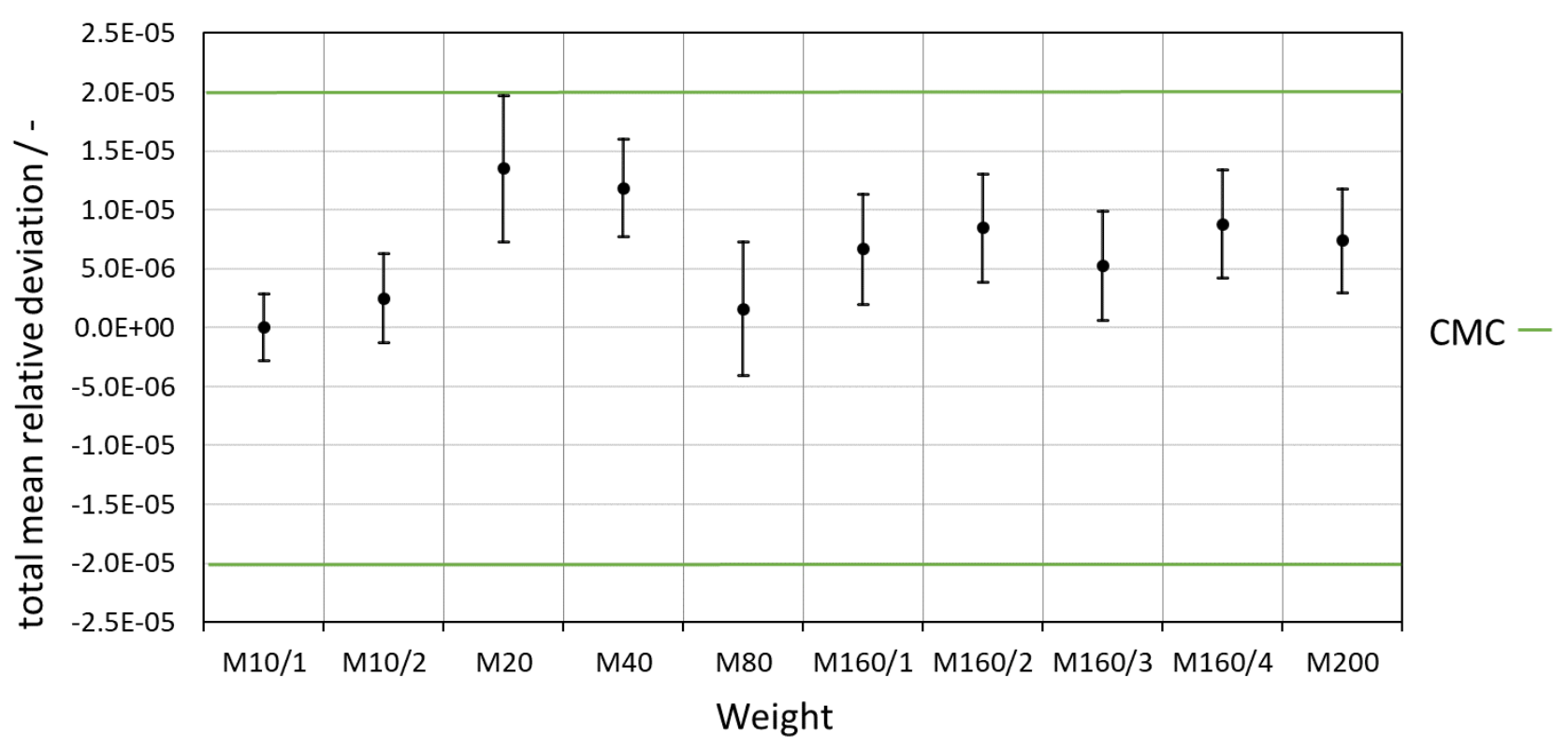

Figure 2: Total mean relative deviations of the $1 \mathrm{MN}$ DFSM weights 


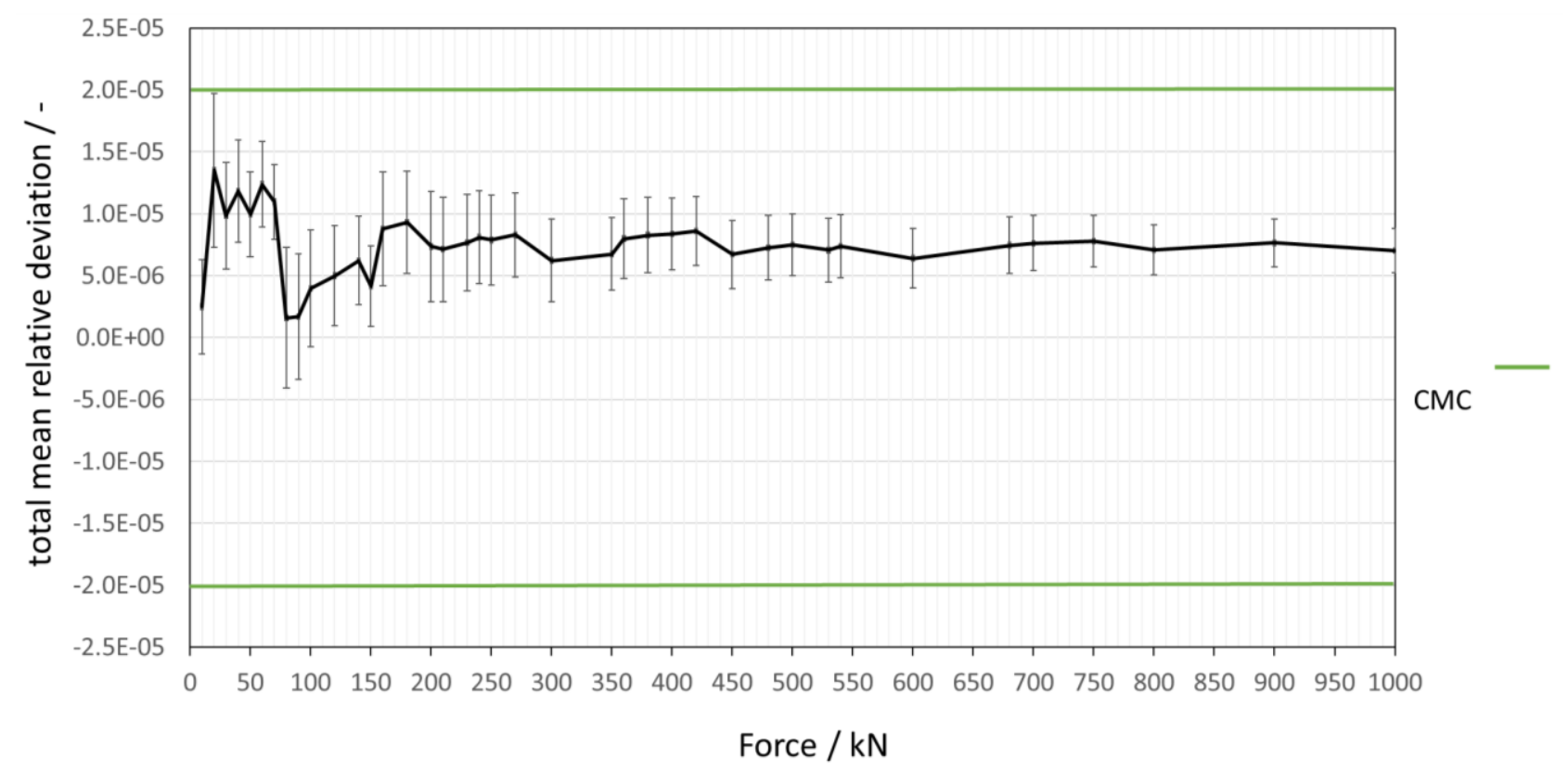

Figure 3: Total mean relative deviations of typical forces generated during ordinary calibration procedures

\section{SUMMARY}

The verification of the INRiM $1 \mathrm{MN}$ deadweight force standard machine was performed with a selfcalibration procedure. The self-calibration method is based on the comparison between two nominally equal forces alternatively generated by a single weight and by an equivalent group of smaller weights. Experimental measurements and the uncertainty budget assessment were performed. Results are within a relative expanded uncertainty of $2 \times 10^{-5}$, i.e. within the limits declared in the CMCs. In the future, the possibility to correct these deviations during ordinary calibration procedures will be investigated.

\section{REFERENCES}

[1] G. Barbato, A. Bray, F. Franceschini, R. Levi, F. Trevissoi, "The new $1 \mathrm{MN}$ dead-weight force standard machine: Description of the installation procedure followed at the Istituto di Metrologia G. Colonnetti”, in Proc. of XIII IMEKO World Congress, pp. 183-188, Torino, Italy, 1994.

[2] A. Germak, L. Marzola, G. Pratola, F. Trevissoi, G. Barbato, "Rapporto tecnico Interno R417 IMGC", Technical report, Torino, Italy, 1995.
[3] Calibration and Measurement Capabilities (CMCs), BIPM. Online [accessed 20200824]: https://www.bipm.org/kcdb/

[4] G. Barbato, A. Germak, "Rapporto tecnico R411", Technical report, INRiM, Torino, Italy, 1995.

[5] C. Ferrero, C. Marinari, E. Martino, "Force metrology in Italy: the new $1 \mathrm{MN}$ deadweight force standard", in Proc. of IMEKO $16^{\text {th }}$ TC3 Conference, Taejon, Korea, 14-18 September 1998.

[6] G. Barbato, F. Trevissoi, P. Iudici, "Pesata delle masse di riferimento della macchina campione di forza da $1 \mathrm{MN}$ ", Technical report, INRiM, Torino, Italy, 1995.

[7] JCGM 100:2008, Evaluation of measurement data Guide to the expression of uncertainty in measurement, Online [accessed 20200825]: https://www.iso.org/sites/JCGM/GUM/JCGM100/C 045315e-html/C045315e.html?csnumber $=50461$

[8] ISO 376:2011- Metallic materials - Calibration of force-proving instruments used for the verification of uniaxial testing machines, ISO, 2011.

[9] R. Kumme, P. Averlant, T. Bartel, A. Germak, A. Knott, J. Man, N. Medina, A. Ostrivnoy, Y. Park, D. Röske, R. Seifarth, M. Wozniak, K. Ueda, Z. Zhimin, "Final report on the force key comparison CCM.F-K3”, Metrologia, vol. 56, 07001, 2019. 